\title{
Treatment of abdominal wall hernia with suture, or polypropylene, or collagen prosthesis ${ }^{1}$
}

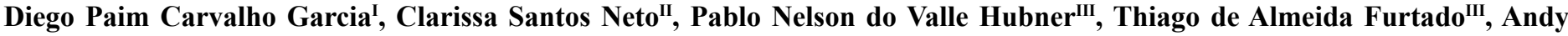 \\ Petroianu $^{\text {Iv }}$, Luiza Ohasi de Figueiredov, Luiz Ronaldo Alberti ${ }^{\mathrm{VI}}$
}

DOI: http://dx.doi.org/10.1590/S0102-865020160060000002

${ }^{\mathrm{I}} \mathrm{PhD}$, Associate Professor, General Surgeon, Department of Surgery, Instituto de Ensino e Pesquisa da Santa Casa, Belo Horizonte-MG, Brazil. Intellectual and scientific content of the study, design the protocol, technical procedures, manuscript writing.

"MD, General and Trauma Surgeon, Hospital Universitário São José, Belo Horizonte-MG, Brazil. Acquisition and interpretation of data, statistical analysis, design the protocol, technical procedures, macroscopic and histopathological examinations.

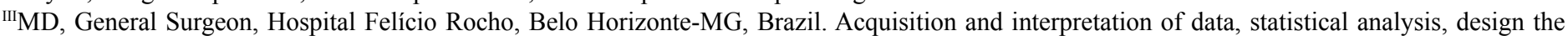
protocol, technical procedures, macroscopic and histopathological examinations.

${ }^{\mathrm{IV}} \mathrm{PhD}$, Full Professor, Department of Surgery, Faculdade de Medicina, Universidade Federal de Minas Gerais (UFMG), Belo Horizonte-MG, Brazil. Intellectual and scientific content of the study, design the protocol, provided guidelines for the surgical interventions, supervised all phases of the study. ${ }^{v}$ Fellow of Surgery, Hospital Felício Rocho, Belo Horizonte-MG, Brazil Acquisition and interpretation of data, statistical analysis, manuscript revision. ${ }^{v} \mathrm{PhD}$, Associate Professor, General Surgeon, Department of Surgery, Faculdade de Medicina, UFMG, and Instituto de Ensino e Pesquisa da Santa Casa, Belo Horizonte-MG, Brazil. Acquisition and interpretation of data, statistical analysis, design the protocol, technical procedures.

\section{ABSTRACT}

PURPOSE: To develop an experimental model for incisional hernias and to compare morphological and functional aspects of hernia repairs by suture, polypropylene mesh and collagen mesh.

METHODS: A defect measuring $7 \mathrm{~cm}$ x $2 \mathrm{~cm}$ was created in the anterior abdominal of 28 New Zealand male rabbits, divided into four groups ( $\mathrm{n}=7)$ : (1) control, (2) suture of the anterior sheath of the rectus abdominal muscle, (3) setting of polypropylene mesh, and (4) setting of collagen mesh. On the 90th postoperative day, the animals were examined to verify the presence of incisional hernia. Samples of abdominal wall and scar were collected for histological study.

RESULTS: Incisional hernia was identified in $85.7 \%$ of the control group, $57.1 \%$ of the suture group, $42.9 \%$ of the collagen mesh group, and none in the polypropylene mesh group $(\mathrm{p}=0.015)$. Mesh exposure could be identified in $71.4 \%$ of the animals in group 3 and in no animal in group $4(\mathrm{p}=0.021)$. The polypropylene mesh is effective in the treatment of abdominal wall defects, causing an intense inflammatory reaction.

CONCLUSION: The collagen mesh is biocompatible, producing a minimal inflammatory reaction, but fails in the treatment of abdominal wall defects.

Key words: Hernia, Ventral. Surgical Mesh. Polypropylenes. Collagen. Rabbits. 


\section{Introduction}

The incisional hernia consists of the protrusion of ofalls, through orifices or abdominal wall, abnormally weakened by congenital defects or traumas and surgical procedures. Suture repair is commonly ineffective, with reports of recurrence in $25 \%$ to $52 \%$ of cases; however, the use of mesh has decreased this recurrence to a rate of less than $10 \%{ }^{1,2}$.

The use of prostheses in fascial-defect repair has resulted in reduction of the recurrence of abdominal wall hernias ${ }^{2,3}$. Recent data from a study on prospective, random, and controlled suture repair versus prosthetic implant revealed much better results when mesh was used ${ }^{4}$. Analysis of a population demonstrated that the prostheses implant, increased from $35 \%$ in 1987 to $66 \%$ in $1999^{5}$. The American Hernia Society defined the use of mesh as a standard procedure in incisional hernia repair ${ }^{6}$. The setting of the mesh allows for the stress-free restoration of the structural integrity of the abdominal wall. Advantages for the use of mesh include its availability, non-dependence on integrity, as well as the resistance of the patient's tissues ${ }^{3,7}$. The ideal material for the implant must be atoxic, non-immunogenic, and non-reactive ${ }^{8,9}$, aimed at using the implant and incorporating it within the surrounding tissues. The risk of infection of the mesh and enteric fistulas limit its use. The development of substances combining non-absorbable, absorbable material and biological materials is of utmost importance in an attempt to reduce the complications related to the mesh and to restore the functions of the abdominal wall ${ }^{11,12}$.

Advances in tissue engineering technology have led to the development of biomaterials derived from human and animal tissues ${ }^{7,12,13}$. These materials differ amongst themselves, as they act through a regenerative process. For those based on collagen, the extra-cellular matrix is preserved, which allows for the maintenance of the mechanical integrity, while supplying a substrate for the regeneration of the host tissue. These materials have shown a resistance to infection, tolerance to cutaneous exposure, and mechanical stability, when used in incisional hernia repair. The disadvantages include their high cost and the lack of long-term comparative studies ${ }^{1,10,11,14-23}$.

The knowledge of the anatomic and physiological structure of the abdominal wall is necessary for success concerning incisional hernia repair ${ }^{18}$. Relapse after mesh repair is rarely caused by an intrinsic flaw in the prosthetic material, as it is more commonly related to the non-identification of the healthy fascia and the poor setting of the mesh, frequently leading to recurrence in the fascia interface with the mesh ${ }^{1,10,11,15}$. In view of this gap in the literature, this present study aimed to employ an experimental incisional hernia model to compare morphofunctional aspects of repairs by means of direct suture, polypropylene or collagen mesh.

\section{Methods}

This work was carried out in accordance with that recommended by the International Standards for the Protection of Animals and the Brazilian Animal Experimentation Code (1988), and was approved by the Committee of Ethics in Animal Experimentation, Universidade Federal University de Minas Gerais, protocol number 098/2011.

Tweenty-eight New Zeland male rabbits, three months of age and with weights of above two kilograms, acquired from the Experimental Veterinary Farm were studied. All of the rabbits were identified and placed in the Biothery of the School of Medicine, one animal per cage. They received rations for rabbits and filtered water ad libitum.

The rabbits were anesthetized with an intramuscular injection in the gluteal region of 5\% ketamine hydrochloride $\left(\right.$ Ketamin-S ${ }^{\circledR}(+)$, Cristália, Itapira-SP) at a dose of $35 \mathrm{mg} / \mathrm{kg}(0.7$ $\mathrm{ml} / \mathrm{kg}$ ), coupled with $2 \%$ xylazine hydrochloride (Rompun ${ }^{\circledR}$, Bayer, Sao Paulo-SP) at a dose of $6 \mathrm{mg} / \mathrm{kg}(0.3 \mathrm{ml} / \mathrm{kg})$. When necessary, half of the initial dose of the anesthesia was applied. During the entire period of anesthesia, the heartbeats and respiration were observed, as were the voluntary movement of the rabbits, in an attempt to detect complications.

After the trichotomy of the abdomen, antisepsis was carried out, using a $2 \%$ degerming chlorhexidine solution followed by a $70 \%$ alcohol and the setting of surgical fields. A defect measuring $7 \mathrm{~cm} \times 2 \mathrm{~cm}$ was created in the ventral abdominal wall with removal of a muscle-aponeurotic sheaf, using single card template for all animals. The defect was closed at the same time. The rabbits were divided into four groups by a random drawing $(n=7)$ :

- Group 1: Control - only a skin suture with no reconstruction of the abdominal wall;

- Group 2: Anterior sheath suture of the abdominal rectus muscle with a continuous 3-0 monofilament polypropylene suture;

- Group 3: Setting of polypropylene mesh, $10 \mathrm{~cm} \mathrm{x} 4$ $\mathrm{cm}$ on the borders of the anterior sheath of the abdominal rectus muscle, with a 3-0 monofilament polypropylene suture (Figure 1A).

- Group 4: Setting of the polymerized and purified type I collagen mesh, $10 \mathrm{~cm} \mathrm{x} 4 \mathrm{~cm}$, on the borders of the anterior sheath of the abdominal rectus muscle, with a 3-0 monofilament polypropylene suture (Figure 1B). 


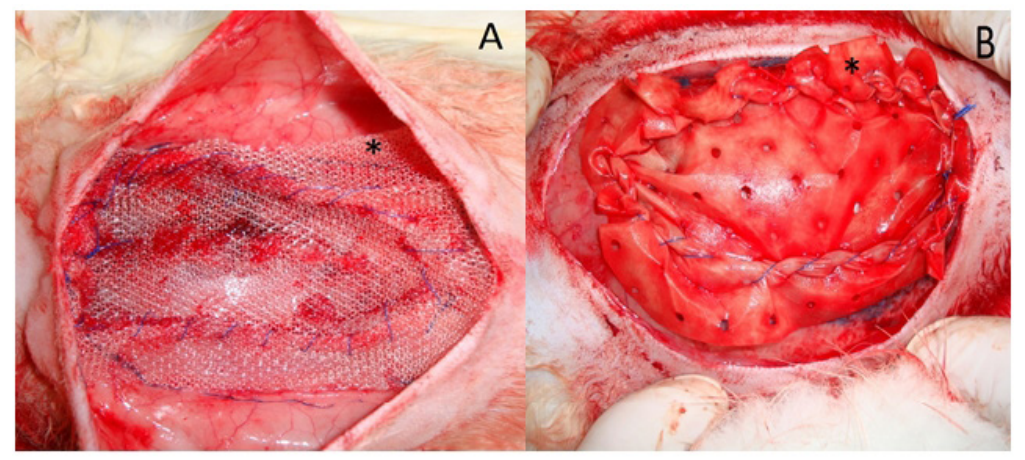

FIGURE 1 - Treatment of incisional abdominal hernia in the rabbit. A - Polypropylene mesh *, $10 \mathrm{~cm} \mathrm{x} 4 \mathrm{~cm}$, sutured on the borders of the abdominal wall defect (Group 3). B - Collagen mesh *, $10 \mathrm{~cm} \mathrm{x} 4 \mathrm{~cm}$, sutured on the borders of the abdominal wall defect (Group 4).

After the surgery and during the entire follow-up period, the rabbits received rations and filtered water ad libitum, and were kept in individual cages, under appropriate conditions of hygiene, ventilation and natural illumination.

Before being killed, the animals were evaluated by means of ectoscopy (aspect of the surgical wound area, with the animal in orthostatism) to verify the presence of incisional hernia.

At the end of the follow-up period, on the 90th postoperatory day, the animals were killed with an intravenous injection of $3 \mathrm{ml}$ of $19.1 \%$ potassium chloride, after an intramuscular injection of $3 \mathrm{ml}$ of ketamine hydrochloride $(50 \mathrm{mg} / \mathrm{ml})$.

A laparotomy in $\mathrm{U}$, released a quadrangular portion of the abdominal wall, to study the incisional hernia and to evaluate the repairs. The removed abdominal wall was then prepared for histological study and stained with hematoxylin and eosin (HE) and Masson trichrome staining. The microscopic evaluation with HE staining was performed to quantify the foreign body gigantic cells, polymorphonuclear (PMN) cells, and mononuclear (MN) cells. Masson's staining was performed in groups 3 and 4, to characterize the collagen fibers in a qualitative manner.

The analyses were performed using $R$, version 2.7.1, and Epi Info, version 6.04, softwares, both of public domains. The variable categorical responses were compared with the overall group by means of contingency tables to which the Fisher exact test was applied. To make a comparison between the weight and the groups, the F test was used (ANOVA), given that the supposition of normality was verified by the Shapiro-Wild test. The comparisons between the two to two groups were performed using the Tukey post hoc method. The values were considered significant for $\mathrm{p}<0.05$.

\section{Results}

All of the animals recovered spontaneously from the surgeries and survived the three-month experiment. The incisional hernia was identified in $100 \%$ of the rabbits from the control group, in $57.1 \%$ from the suture repair group, and in $42.9 \%$ from the biological mesh group (Table 1). No rabbit from the polypropylene mesh presented incisional hernia $(\mathrm{p}=0.015)$.

No difference in the presence of abscesses among the groups could be observed. Mesh exposure could be identified in $71.4 \%$ of the animals from group 3 and in no animal from group 4 $(\mathrm{p}=0.021)$. Therefore, it could be observed that mesh exposure and the group are in fact associated, with the rabbits which received a polypropylene mesh closure exhibiting a greater chance of presenting mesh exposure than those which received the biological mesh closure (Table 1).

TABLE 1 - Comparison among the control, suture repair, polypropylene mesh, and collagen mesh groups concerning the percentage of the incidence of mesh exposure and incisional hernia.

\begin{tabular}{|c|c|c|}
\hline \multicolumn{3}{|c|}{$\begin{array}{l}\text { Percentage of incidence of mesh exposure and incisional } \\
\text { hernia }\end{array}$} \\
\hline & Mesh exposure & Incisional hernia \\
\hline Control & - & $100 \%$ \\
\hline Suture repair & - & $57.1 \%$ \\
\hline Polypropylene mesh & $71.4 \%{ }^{* 2}$ & $0 \% * 1$ \\
\hline Collagen mesh & $0 \%$ & $42.9 \%$ \\
\hline
\end{tabular}

${ }^{* 1} \mathrm{p}=0.015 ;{ }^{* 2} \mathrm{p}=0.021$

The present study highlighted a greater proportion of rabbits from the control group with a zero count in the average PMN number (100\%), as well as greater proportions from the suture repair, polypropylene mesh, and biological mesh groups concerning PMN numbers with average counts of higher than 10 ( $71.4 \%, 71.4 \%$, and $57.1 \%$, respectively) $(\mathrm{p}=0.043)$. All of the rabbits from the control and polypropylene mesh groups presented more than 10 monocytes. By contrast, all of the rabbits from the suture groups presented from 1 to 5 monocytes, while $28.6 \%$ of 
the rabbits from the biological mesh closure group presented from 1 to 5 monocytes, and $71.4 \%$ presented more than 10 monocytes $(p<0.001)$. Finally, the rabbits from the control, suture repair, and biological mesh groups presented no foreign body gigantic cells, while the rabbits of the polypropylene mesh group presented more than 10 foreign body gigantic cells (Table 2).

TABLE 2 - Comparison among the control, suture repair, polypropylene mesh, and collagen mesh groups concerning the percentage of the incidence of polymorphonuclear monocytes and foreign body gigantic cells.

Percentage of the incidence of polymorphonuclear, monocytes and foreign body gigantic cells

\begin{tabular}{|c|c|c|c|c|c|c|c|c|c|}
\hline \multirow[b]{2}{*}{ Average number of cells } & \multicolumn{3}{|c|}{ Polymorphonuclear *1 } & \multicolumn{3}{|c|}{ Monocytes *2 } & \multicolumn{3}{|c|}{ Foreign body gigantic cell ${ }^{* 2}$} \\
\hline & 0 & $1-5$ & $>10$ & 0 & $1-5$ & $>10$ & 0 & $1-5$ & $>10$ \\
\hline Control & $100 \%$ & - & - & - & - & $100 \%$ & $100 \%$ & - & - \\
\hline Suture repair & - & $28.6 \%$ & $71.4 \%$ & - & $100 \%$ & - & $100 \%$ & - & - \\
\hline Polypropylene mesh & $28.6 \%$ & - & $71.4 \%$ & - & - & $100 \%$ & - & - & $100 \% *$ \\
\hline Collagen mesh & - & $42.9 \%$ & $57.1 \%$ & - & $28.6 \%$ & $71.4 \%$ & $100 \%$ & - & - \\
\hline
\end{tabular}

${ }^{* 1} \mathrm{p}=0.043 ;{ }^{* 2} \mathrm{p}<0.001$

In the collagen mesh group, a more homogeneous aspect could be observed, associated with a more ordered architectural arrangement of the collagen fibers, possibly stemming from the manner in which they were created. In the polypropylene mesh group, the gigantic cell reaction led to a diffuse architectural distortion of the collagen fiber pattern, as compared to the normally observed architecture, alterations which also occurred in the animals with abscess in their wounds (Figure 2).

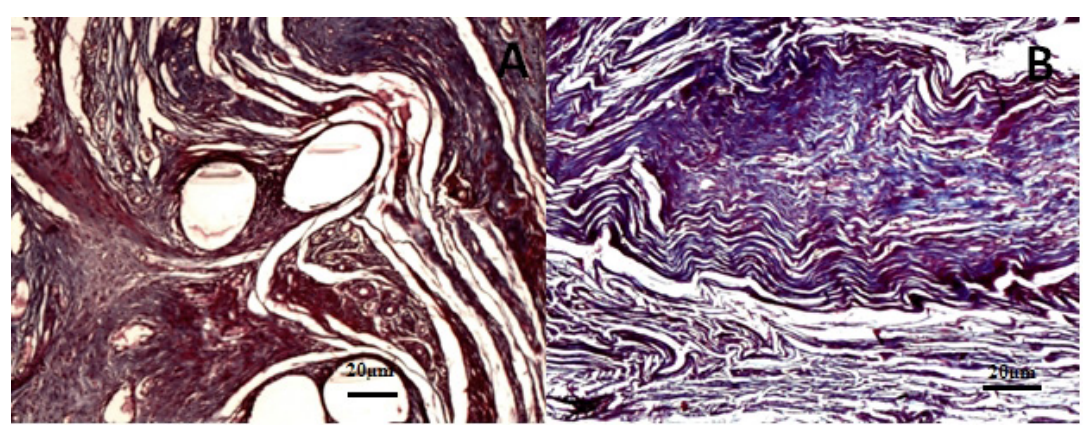

FIGURE 2 - Photomicrograph of A - group 3- polypropylene mesh and B - group 4 - collagen mesh, stained with Masson trichrome (x100). Observe the more homogeneous aspect associated with a more ordered architectural arrangement of the collagenous fibers (collagen mesh) and gigantic cell reaction with a diffuse architectural distortion of the collagen fiber standard regarding the observed architecture (polypropylene mesh).

\section{Discussion}

The animals used in this work were rabbits, concerning the observation of their anatomy and feasibility of the surgical procedure without the need for special materials, in addition to the lower degree of complexity in caring for the animals during the entire post-operatory period ${ }^{11,12}$. The formulated experimental model, represented by group 1, presented an incisional hernia in $100 \%$ of the animals, demonstrating an effective model of abdominal wall hernia.

In this study, the mesh exposure presented as a complication of the surgical site. The animals from group 3 presented a polypropylene mesh exposure in five cases $(71.4 \%)$, which did not occur in the other groups $(p<0.005)$. However, none of the animals from group 3 presented an incisional hernia, with the secondary mesh exposure mainly representing the intense inflammatory reaction of the organism against the implant, without being associated, in this study, with the increase in the incidence of hernias ${ }^{17}$.

The polypropylene mesh proved to be effective in abdominal wall repair, given that no animal from group 3 developed an incisional hernia, confirming the use of implants, more specifically polypropylene mesh, in the treatment of incisional hernias as a standard procedure ${ }^{2,4,8,10,12}$. No statistically significant difference among the other groups could be observed regarding the incisional hernia.

The biological mesh studied herein presented $42.9 \%$ of failure hernia repair. One factor that may well have collaborated in 
bringing about this result was the use of the inlay technique for the setting of the mesh ${ }^{16}$. Jin et al. ${ }^{10}$ demonstrated that the relapse was reduced when the separation of the components was combined with the setting of the biological implant and, inversely, when the biological mesh was set as a bridge (inlay), without bringing the borders of the hernia orifice closer together (recurrence rate of $80 \%$ ). The Ventral Hernia Working Group (VHWG) recommend that the majority of biological implants must be implanted under the proper stress to prevent the further loosening of the material, as well as with different inlay techniques ${ }^{13,14}$.

In the present study, the intensity of the inflammatory reactionwasevaluatedbytheaveragecountofthepolymorphonuclear cells, monocytes, and foreign body gigantic cells ${ }^{11}$. As expected, all groups presented some degree of inflammatory reaction; however, an intense reaction of the foreign body could be identified in group 3 , represented by the average count of foreign body gigantic cells, presenting a statistically significant difference as compared to the other groups. The polypropylene implant caused an intense foreign body reaction. This microscopic result was in accordance with macroscopic findings regarding the mesh exposure $(p<0.05)$ and may well explain the absence of incisional hernias in this group. The inflammatory reaction of the host against the polypropylene implant produced a fibrous and rigid scar, hindering herniation; however, the continuous activation caused by the presence of the foreign body led to the chronicity of this inflammatory process, in turn causing the exposure of the mesh in an attempt to eliminate the organism $^{10,14}$. Mesh exposure might be related with the thickness of subjects' abdominal wall, however such intense inflammatory and foreign body reaction, causing mesh extrusion, did not occur with the use of type I collagen mesh. Thus. indicating a stronger response due to type of mesh material rather than the choice of research subjects.

By contrast, it could be observed that the type I collagen mesh behaved similarly to the host tissue by not unleashing an inflammatory reaction upon the foreign body $y^{3,7,17}$. In addition, it was impossible, by optical microscopy and by the methods used in this study, to differentiate between the collagen of the biological implant and that of the host. This can be explained by the fact that the mesh collagen was substituted by the host collagen both partially and completely or that it had been reabsorbed. This differentiation is important to verify whether or not the mesh had been identified as "self" or "not-self"15, which may well explain the observed failures ${ }^{3,14,17,21}$. If the mesh is identified by the host as a "self", regeneration and integration of the mesh to the original tissue occurs, demonstrating that the mesh is biocompatible, but needs a greater tensile strength, be it by increasing the number of layers of collagen or by using the cross linking method. If it is identified as "not-self", the mesh would be reabsorbed with a reduction in tensile strength before the complete healing and relapse of the hernia. Accordingly, cross linking can also be used to hinder the reabsorption of the mesh, increasing the time for the growth of the host tissue and producing a more resistant healing process.

This study, using animal as main subjects, shows that polypropylene mesh are effective regarding the correction of abdominal wall defects and prevention of hernia recurrences, as seen in humans. The same cannot be said about type I collagen mesh, since this material was not used in studies with humans. Nevertheless, the results might be used as an indication of material behavior in humans when combined for better outcomes, such as in collagen coated polypropylene implants. Future studies are required to evaluate this matter.

\section{Conclusions}

The polypropylene mesh is effective in the treatment of abdominal wall defects, but causing an intense foreign body inflammatory reaction. The collagen mesh is biocompatible, causing a minimum inflammatory reaction, but it was not effective in the treatment of incisional hernias.

\section{References}

1. Shell IV DH, de la Torre J, Andrades P, Vasconez LO. Open repair of ventral incisional hernias. Surg Clin North Am. 2008 Feb;88(1):6183. PMID: 18267162 .

2. Helgstrand F, Rosenberg J, Kehlet H, Jorgensen LN, Bisgaard T. Nationwide prospective study of outcomes after elective incisional hernia repair. J Am Coll Surg. 2013 Feb;216(2):217-28. PMID: 23219350 .

3. Harris HW. Clinical outcomes of biologic mesh: where do we stand? Surg Clin North Am. 2013 Oct;93(5):1217-25. PMID: 24035084.

4. Luijendijk RW, Hop WC, van den Tol MP, de Lange DC, Braaksma MM, Ijzermans JN, Boelhouwer RU, de Vries BC, Salu MK, Wereldsma JC, Bruijninckx CM, Jeekel J. A comparison of suture repair with mesh repair for incisional hernia. N Engl J Med. 2000 Aug;343(6):392-8. PMID: 10933738.

5. Flum DR, Horvath K, Koepsell T. Have outcomes of incisional hernia repair improved with time? A population-based analysis. Ann Surg. 2003 Jan;237(1):129-35. PMID: 12496540

6. Voeller GR, Ramshaw B, Park AE, Heniford BT. Incisional hernia. J Am Coll Surg. 1999 Dec;189(6):635-7. PMID: 10589602.

7. Lukasiewicz A, Skopinska-Wisiniewska J, Marszalek A, Molski S, Drewa T. Collagen/Polypropilene composite mesh biocompatibility in abdominal wall reconstruction. Plast Reconst Surg. 2013 May;131(5):731-40. PMID: 23629112.

8. Grevious MA, Cohen M, Jean-Pierre F, Herrmann GE. The use of prosthetics in abdominal wall reconstruction. Clin Plast Surg. 2006 Apr;33:181-97. PMID: 16638462. 
9. Gao Y, Liu LJ, Blatnik JA, Krpata DM, Anderson JM, Criss NC, Posielski N, Novitsky YW. Methodology of fibroblast and mesenchymal stem cell coating of surgical meshes: a pilot analysis. J Biomed Mater Res B Appl Biomater. 2014 May;102(4):797-805. PMID: 24142485.

10. Bachman S, Ramshaw B. Prosthetic material in ventral hernia repair: how do i choose? Surg Clin North Am. 2008 Feb;88(1):10112. PMID: 18267164.

11. Penttinen R, Grönroos JM. Mesh repair of common abdominal hernias: a review on experimental and clinical studies. Hernia. 2008 Aug;12(4):337-44. PMID: 18351432.

12. Aramayo AL, Lopes Filho GJ, Barbosa CA, Amaral VF, Costa LA. Abdominal wall healing in incisional hernia using different biomaterials in rabbits. Acta Cir Bras. 2013 Apr;28(4):307-16. PMID: 23568239.

13. Ladurner R, Drosse I, Chiapponi C, Bürklein D, Jansson V, Kokott A, Hoffmann B, Ziegler G, Mustchler W, Mussack T, Schieker M. Polypropylene meshes coated with a polysaccharide based bioadhesive for intra-abdominal mesh fixation in a rabbit model. Surg Endosc. 2013 Jun;27(6):1991-6. PMID: 23299133.

14. Breuing K, Butler CE, Ferzoco S, Franz M, Hultman CS, Kilbridge JF, Rosen M, Silverman RP, Vargo D. Incisional ventral hernias: review of the literature and recommendations regarding the grading and technique of repair. Surgery. 2010 Sep;148(3):544-58. PMID: 20304452.

15. Jansen PL, Mertens PR, Klinge U, Schumpelick VS. The biology of hernia formation. Surgery. 2004 Jul;136(1):1-4. PMID: 15232531.

16. Zuvela M, Galun D, Djuric-Stefanovic A, Palibrb I, Petrovic M, Milicevic M. Central rupture and bulging of low-weight polypropylene mesh following recurrent incisional sublay hernioplasty. Hernia. 2014 Feb;18(1):135-40. PMID: 24309998.

17. Lee L, Mata J, Landry T, Khwaja KA, Vassiliou MC, Feldman LS. A systematic review of synthetic and biologic materials for abdominal wall reinforcement in contaminated fields. Surg Endosc. 2014 Sep;28(9):2531-46. PMID: 24619334.

18. Jensen KK, Kiaer M, Jorgensen LN. Abdominal muscle function and incisional hernia: a systematic review. Hernia. 2014 Aug;18(4):4186. PMID: 24728836.

19. Jin J, Rosen MJ, Blatnik J, McGee MF, Williams CP, Marks J, Ponsky J. Use of acellular dermal matrix for complicated ventral hernia repair: does technique affect outcomes? J Am Col Surg. 2007 Nov;205(5):654-60. PMID: 17964441.
20. Montes JHM, Bigolin AV, Baú R, Nicola R, Grossi JVM, Loureiro CJ, Cavazzola LT. Analysis of adhesions resulted from mesh fixation with fibrina sealant and suture - experimental model. Rev Col Bras Cir. 2012 Dec;39(6):509-14. PMID: 23348648.

21. Lamber B, Grossi JVM, Manna BB, Montes JHM, Bigolin AV, Cavazzola LT. May polyester with collagen coating mesh decrease the rate of intraperitoneal adhesions in incisional hernia repair? Arq Bras Cir Dig. 2013 Jan-Mar;26(1):13-7. PMID: 23702864.

22. Kist C, Manna BB, Montes JHM, Bigolin AV, Grossi JVM, Cavazzola. Comparative study of intraperitoneal adhesions associated with the use os meshes of polypropylene and polypropylene coated with omega-3 fatty acid. Rev Col Bras Cir. 2012 May-Jun;39(3):201-6. PMID: 2283656 .

23. Lontra MB, Bigolin AV, Costa RG, Grossi NV, Scalco P, Roll S, Cavazzola LT. Effectiveness of the combined use of lactic acid film and polypropylene mesh in the formation of intraperitoneal adhesions - an experimental modelin rats. Rev Col Bras Cir. 2010 Oct;37(5):364-9. PMID: 21181003.

\section{Correspondence:}

Diego Paim Carvalho Garcia

Rua dos Timbiras, 3642/sala 704

30140-062 Belo Horizonte - MG Brasil

Tel.: (55 31)3295-3411

diegopcg25@hotmail.com

Received: Feb 15, 2016

Review: Apr 18, 2016

Accepted: May 19, 2016

Conflict of interest: none

Financial sources: CNPq and FAPEMIG

${ }^{1}$ Research performed at Laboratory of Experimental Surgery, Department of Surgery, School of Medicine, Universidade Federal de Minas Gerais (UFMG), Belo Horizonte-MG, Brazil. 